\title{
Elaboração e validação de escala de avaliação da sede no paciente cirúrgico: nota prévia
}

\section{Development and validation of a thirst scale for the surgical patient: a preliminary note}

\author{
Patricia Aroni $^{1}$; Renata Perfeito Ribeiro ${ }^{2}$; Lígia Fahl Fonseca ${ }^{3}$
}

\begin{abstract}
Resumo
A sede é um desconforto real relatado por grande parte dos pacientes no pós-operatório imediato, quando ainda permanecem em jejum. Esta nota prévia tem como objetivo o relato da elaboração de uma escala para avaliação da sede do paciente em perioperatório. Pesquisa metodológica, baseada na teoria de elaboração de instrumental psicológico, através dos pólos teórico, metodológico e analítico. Através de pesquisas bibliográficas foi elencado como construto: a sede no paciente cirúrgico, sendo escolhido sete itens que melhor descrevessem a sede: boca seca, ressecamento dos lábios, língua seca, vontade de engolir, falta de saliva, saliva grossa e garganta seca. As próximas etapas da validação da escala se encontram em desenvolvimento. Almeja-se proporcionar à equipe maneiras de avaliar e quantificar a sede do paciente perioperatório.
\end{abstract}

Palavras-chave: Sede. Enfermagem perioperatória. Escala. Estudos de validação.

\begin{abstract}
Thirst is a real discomfort reported by most patients in the immediate postoperative period, when they remain fasting. This preliminary note aims to report the development of a scale to assess thirst in the perioperative patient. Methodological research, based on the theory of psychological instrument development, within the theoretical, methodological and analytical poles. The study was approved by the Ethics Committee of Londrina State University-UEL under CAAE: 02299412.6.0000.5231. After extensive literature research, thirst in the surgical patient was defined as the construct of this study, with seven describing items: dry mouth, dryness of the lips, dry tongue, desire to swallow, lack of saliva, thick saliva and dry throat. The next steps of the scale validation are under development. This study aims to provide ways for the team to assess and quantify thirst of the perioperative patient.
\end{abstract}

Keywords: Thirst. Perioperative nursing. Scale. Validation studies.

\footnotetext{
${ }^{1}$ Enfermeira. Especialista em Enfermagem Perioperatória, Professora Colaboradora, Departamento de Enfermagem da Universidade Estadual de Londrina-PR.

${ }^{2}$ Doutora em Enfermagem, Professora Adjunta do Departamento de Enfermagem, e do Programa de Mestrado em Enfermagem do Departamento de Enfermagem da Universidade estadual de Londrina. Coordenadora do NUESTUEL. Londrina-PR.

${ }^{3}$ Doutora em Enfermagem. Professora Adjunta do Departamento de Enfermagem, área de Enfermagem Perioperatória, Centro Cirúrgico e Central de Material, Coordenadora do GPENP, Londrina-PR.
} 


\section{Introdução}

Dor, dificuldade respiratória, náuseas e vômitos no pós-operatório imediato (POI) têm sido estudados extensivamente com o propósito de serem minimizados através de protocolos e escalas que proporcionem ao paciente segurança no cuidado prestado. A sede, no entanto, tem sido pouco avaliada, mesmo se apresentando como um desconforto real e de alta incidência, oscilando entre $43,8 \%$ a $75 \%$ dos pacientes cirúrgicos que permanecem em jejum (ARONI; NASCIMENTO; FONSECA, 2012). Relatos de pacientes que vivenciaram esse desconforto mostram o quanto é desagradável e difícil vivenciar o sintoma sede ocasionando sentimentos como ansiedade, irritabilidade e desespero (GOIS et al., 2012).

A sede se caracteriza como um sintoma, sendo ele uma experiência subjetiva que engloba alterações biopsicossociais na sensação ou cognição de um indivíduo. Na perspectiva da Teoria do Manejo de Sintomas, a experiência vivenciada é avaliada sob três domínios: a percepção do indivíduo perante o sintoma, a avaliação do seu significado e sua resposta. Dentro da avaliação do seu significado está a percepção e o julgamento do indivíduo sobre a gravidade, tratamento e o efeito dos sintomas em sua vida (HUMPHREYS et al., 2008).

A sede é uma resposta do organismo à falta de água ou ao aumento da concentração osmótica no plasma, classificada como sede hipovolêmica ou sede osmótica respectivamente. A sede hipovolêmica é deflagrada por perdas de pelo menos $10 \%$ do volume plasmático, sendo essas perdas ocasionadas principalmente por hemorragias e vômitos. Por outro lado, a sede osmótica é iniciada com alterações de 1\% a 2\% na osmolaridade plasmática (ARAI; STOTTS; PUNTILLO, 2013). Esses estímulos são captados por diversos receptores centrais e periféricos que enviam mensagens ao sistema nervoso central o qual impulsiona o organismo à busca por água a fim de normalizar todo o processo desencadeador da sede (LEIPER, 2005).
Observa-se na prática que a equipe pouco valoriza o relato de sede do paciente, desconhecendo maneiras seguras de mensurá-la e tratá-la, atendose a procedimentos rotineiros de cuidado sem evidências científicas. Para possibilitar o manejo seguro da sede no paciente cirúrgico, um Protocolo de Segurança para o Manejo da Sede (PSMS) foi desenvolvido, tendo como foco a avaliação do nível de consciência, proteção de vias aéreas e ausência de náuseas e vômitos, como indicadores de segurança na administração de métodos de alívio da sede (NASCIMENTO; FONSECA, 2013).

Para que se proporcione um manejo adequado da sede no paciente perioperatório é necessário antes de proporcionar métodos de alívio, avaliar e mensurar essa sede. Estudos avaliando estratégias para a redução da sede englobam desde a utilização de aplicações de gaze molhada, umidificação da cavidade oral, realização de gargarejos com água fria, solução salina gelada, aplicação de gelo e gaze úmida, o uso de água em temperatura ambiente e uso de lascas de gelo (ARONI; NASCIMENTO; FONSECA, 2012; CHO; KIM; PARK, 2010; HUR et al., 2009; YOON; MIN, 2011).

A percepção da sede envolve complexos mecanismos regulatórios fisiológicos assim como é influenciada por questões ambientais, culturais de cada indivíduo. Dessa forma, avaliar e mensurar a sede é um desafio de grande escopo. Diferentes métodos têm sido utilizados na busca de definir o grau e intensidade para diferentes populações, dentre eles, o uso da escala visual analógica a qual se mostrou capaz de identificar a intensidade da sede percebida pelo paciente quantificando-a (ARONI; NASCIMENTO; FONSECA, 2012; MILLARDSTAFFORD et al., 2012; ROLLS et al., 1980), a análise laboratorial da osmolaridade plasmática relacionando-a com a percepção da sede (ROLLS et al., 1980) e o uso de aparelhos de ressonância magnética funcional e de tomografia com emissão de pósitron para a verificação de áreas cerebrais relacionadas ao sentimento de sede (LEIPER, 2005; DENTON et al., 1999). 
A única escala de avaliação do desconforto da sede validada foi desenvolvida para pacientes em tratamento de hemodiálise ambulatorial, onde é necessário um controle rigoroso da ingesta hídrica. Essa escala contempla três domínios: duração, frequência e desconforto, sendo necessário que o paciente se encontre em total domínio de suas percepções, fato que não abrange o paciente cirúrgico, pois o mesmo se encontra em momento de recuperação anestésica (WELCH, 2002).

Tradicionalmente utiliza-se uma escala visual numérica para quantificar a intensidade da sede, contudo, na prática clinica se verifica a necessidade de uma escala de sede específica para o paciente cirúrgico, que contemplem seus sinais e que verificando de forma eficaz e auxiliando no manejo adequado deste sintoma. Sendo assim, esta nota prévia tem como objetivo o relato da elaboração de uma escala para avaliação da sede do paciente em perioperatório.

\section{Método}

Estudo metodológico, onde o método se baseia na teoria de elaboração de instrumental psicológico, o qual compreende três grandes polos: teórico, empírico ou experimental e analítico. O pólo teórico compreende seis passos que constituem desde a teorização do construto à elaboração do instrumento. O primeiro passo compreende o sistema psicológico onde o pesquisador deve saber o que ele quer trabalhar, fundamentando seu construto. No segundo passo deve-se limitar o objeto de estudo definindo os aspectos específicos que se deseja estudar e as propriedades que serão objeto da pesquisa. Após as definições, no terceiro passo ocorre a categorização do construto em unifatorial ou multifatorial, sendo definidas de acordo com os fatores que distinguem o construto (PASQUALI, 1999).

O quarto passo do pólo teórico consiste na enumeração de itens que melhor descrevam o construto, além da definição constitutiva e operacional dos mesmos. A definição constitutiva referencia a definição de um construto por meio de outros construtos, sendo esta definição a que tipicamente aparece nos dicionários e enciclopédias. Por outro lado, a definição operacional é quando o item é definido em termos de operações concretas, de comportamentos físicos que o expressam (PASQUALI, 1999).

O quinto passo compreende a operacionalização do construto e a elencação de itens que o melhor descreva. Os itens podem ser extraídos de várias fontes como a entrevista com a população para a qual se pretende construir o instrumento, literaturas referentes ao construto em estudo e experiências vivenciadas pelos pesquisadores. Os itens são construídos para representar comportamentalmente o construto de interesse (PASQUALI, 1999).

No passo seguinte ocorre a análise semântica dos itens juntamente com a análise dos juízes. Na análise semântica deve-se objetivar a compreensão dos itens por toda a população pela qual o instrumento será utilizado. Assim, através da análise do instrumento com o extrato mais baixo da população almeja-se o entendimento do mesmo por todos os indivíduos, enquanto que a análise do instrumento por uma amostra mais sofisticada deve ser realizada afim de evitar deselegância na formulação dos itens. Se houver divergência na reprodução dos itens ou ser entendido diferentemente do que o pesquisador quis elucidar, o mesmo deverá ser reformulado e reapresentado até que não ofereça dificuldade nenhuma de compreensão (PASQUALI, 1999).

A próxima etapa, ainda no sexto passo, é a análise dos juízes conhecida também como análise de construto. Nesta etapa, os juízes fazem a verificação de cada item elencando os que estejam melhor estruturados e avaliando a sua real ligação com o propósito do instrumento (PASQUALI, 1999).

O próximo procedimento se refere ao pólo experimental onde consiste a fase de validação do instrumento piloto, abrangendo dois passos: o planejamento da aplicação e a própria coleta da 
informação. Na etapa de planejamento, deve-se definir a amostra juntamente com o esclarecimento de instruções de como aplicar o instrumento sendo que, na classificação da amostra deve-se definir a população para a qual o instrumento será aplicado, além da quantidade mínima de indivíduos para que o instrumento passe a ser fidedigno ao restante da população (PASQUALI, 1999).

No passo seguinte onde se prioriza a coleta de informação, o pesquisador deve elucidar com clareza os objetivos do instrumento tendo em vista as formas de aplicação do mesmo, podendo ser de forma coletiva ou individual. Nesta etapa também deve-se esclarecer como será a resposta do indivíduo, se será em forma de alternativas, escolha forçada, escala de intensidades entre outros (PASQUALI, 1999).

O terceiro e último pólo, consiste nos procedimentos analíticos. Esta etapa compreende os quatro últimos passos para a validação e normatização do instrumento e para que isso ocorra, primeiramente deve-se definir a dimensionalidade do mesmo de acordo com uma análise fatorial. O instrumento é elaborado pelo autor de forma empírica firmando a hipótese de único fator, contudo há necessidade de ser demonstrado com o auxílio da análise fatorial, quantos fatores mede, além da demonstração da própria validade do instrumento juntamente com a análise preliminar dos itens (PASQUALI, 1999).

Ainda no terceiro polo, o próximo passo abrange a análise empírica que consiste em verificar as características que itens do instrumento devem apresentar. No terceiro passo busca-se avaliar a precisão de escala de acordo com a correlação entre variância verdadeira e variância erro, sendo que quanto maior a primeira e menor a segunda, mais fidedigno é o instrumento. Existem várias técnicas para verificar a precisão de um teste, entre elas o teste-reteste, formas paralelas e a consistência interna (PASQUALI, 1999).

Ao passar pelos passos descritos, o instrumento pode ser considerado válido e fidedigno, estando pronto para o uso em pesquisa. Contudo, se para o uso em técnica clínica o instrumento será submetido à normatização a fim de interpretar os resultados produzidos, sendo este o último passo para construção, validação e posteriormente uso na população alvo (PASQUALI, 1999).

O projeto de pesquisa foi aprovado pelo Comitê de Ética da Universidade Estadual de Londrina- UEL sob o CAAE: 02299412.6.0000.5231.

\section{Resultados e Resultados Esperados}

De acordo com as etapas referentes à teoria de construção de escalas, alguns passos já foram alcançados neste estudo. No pólo teórico, o primeiro passo foi alcançado sendo realizada através de revisões bibliográficas a fundamentação do construto elencado, a sede no paciente cirúrgico.

No parâmetro da multifatorialidade do construto da sede no paciente cirúrgico, buscouse através de literatura pertinente, da observação e experiência clínica dos autores além de entrevistas semi-estruturadas, itens que melhor descrevam o construto. Após avaliações sobre esses itens chegouse a um total de sete itens que melhor avaliassem a sede, são eles: boca seca, ressecamento dos lábios, língua seca, vontade de engolir, falta de saliva, saliva grossa e garganta seca.

Seguindo o pólo teórico o próximo passo consiste na definição constitutiva e definição operacional (PASQUALI, 1999). Na definição constitutiva, os itens: boca seca, língua seca e garganta seca foram elucidados como ausência ou produção insuficiente de saliva ocasionando a secura desses locais e a excitação de receptores orais que levam o indivíduo a sentir sede (GAYTON; HALL, 2002). O ressecamento dos lábios foi definido como a ausência da hidratação das mucosas labiais apresentando fissuras e crostas sendo esse sinal resultado da própria desidratação causada pelo jejum prolongado mas potencializado pelo uso de algumas drogas anestésicas como a atropina e alguns procedimentos anestésicos-cirúrgicos 
como a intubação (GAYTON; HALL, 2002). O item vontade de engolir foi descrito como o desejo incontrolável de umidificação da mucosa oral a fim de normalizar todos os processos desencadeadores da sede. Por outro lado, os itens falta de saliva e saliva grossa foram definidos como ausência da saliva e modificações em sua composição respectivamente, sendo causados pela diminuição do reflexo de deglutição, diminuição da produção de saliva e constante abertura de cavidade oral em anestesia geral (GAYTON; HALL, 2002).

$\mathrm{Na}$ definição operacional foram elaborados cinco tópicos para posterior construção do instrumento piloto. O primeiro tópico abrange questões de avaliação da boca, língua e garganta questionando o paciente sobre a secura desses locais. O segundo tópico é composto por uma inspeção criteriosa de mucosa labial com o objetivo de classifica-la em: úmida e sem fissuras, ressecada ou com fissuras e crostas. Seguindo a construção do instrumento temse o terceiro tópico que questionará a hipossalivação e alteração da viscosidade salivar, seguida pelo quarto e quinto tópico onde avaliará a vontade do indivíduo de beber algum líquido e a vontade dele beber água respectivamente.

As próximas etapas deste estudo ainda estão em fase de desenvolvimento. A primeira será a realização de análise semântica que consiste em avaliar os itens pelo substrato mais baixo da população que usará o instrumento, sendo representada pelos técnicos de enfermagem que trabalham na sala de recuperação e após, será avaliado por indivíduos do substrato mais alto, enfermeiros, com o objetivo dos mesmos avaliarem quanto à sua apresentação.

Após o termino da análise semântica os itens elencados passarão para a análise de juízes, onde serão selecionados experts no assunto para a realização de uma análise teórica dos mesmos. A seleção experts será composta por médicos anestesistas e enfermeiros especialistas em Sala de Recuperação Anestésica. Nesta pesquisa, a concordância dos especialistas quanto ao significado dos itens deverão alcançar no mínimo $80 \%$, sendo esta porcentagem estabelecida como segura para se alcançar a fidedignidade da pesquisa (BERTONCELLO, 2004; WESTMORELAND et al., 2000).

As etapas seguintes que compreendem o pólo experimental e o pólo analítico, estão em fase de desenvolvimento.

\section{Conclusão}

O paciente cirúrgico pertence a um grupo de risco para o desenvolvimento do sintoma sede devido aos procedimentos e condutas realizadas no perioperatório. Com esse estudo pretende-se aplicar de forma segura e eficaz uma escala de avaliação da sede específica para este paciente sendo que sua aplicabilidade norteará os cuidados a serem ministrados ao paciente com sede proporcionando segurança e qualidade no seu manejo.

\section{Referências}

ARAI, S.; STOTTS, N.; PUNTILlO, K. Thirst in critically ill patients: from physiology to sensation. American Journal of Critical Care, Aliso Viejo, v. 22, n. 4, p. 328-35, 2013. Disponível em: <http://www.ncbi.nlm.nih.gov/ pubmed/23817822>. Acesso em: 5 abr. 2014.

ARONI, P.; NASCIMENTO, L. A.; FONSECA, L. F. Avaliação de estratégias no manejo da sede na sala de recuperação pós-anestésica. Acta Paulista de Enfermagem, São Paulo, v. 25, n. 4, p. 530-6, 2012. Disponível em: <http://www.scielo. br/pdf/ape/v25n4/en_08.pdf $>$. Acesso em: 5 abr. 2014.

BERTONCELLO, K. C. G. Qualidade de vida e a satisfação da comunicação do paciente após laringectomia total: construção e validação de um instrumento de medida. 2004 . Tese (Doutorado em Enfermagem Fundamental) - Escola de Enfermagem de Ribeirão Preto, Universidade São Paulo, Ribeirão Preto, 2004. 
CHO, E. A.; KIM, K. H.; PARK, J. Y. Effects of frozen gauze with normal saline and ice on thirst and oral condition of laparoscopic cholecystectomy patients: pilot study. Journal of Korean Academy of Nursing, Seoul, v. 40, n. 5, p. 714-23, 2010.

DENTON, D.; SHADE, R.; ZAMATIPPA, F.; EGAN, G.; BLAIR-WEST, J.; McKINLEY, M.; LANCASTER, J.; FOX, P. Neuroimaging of genesis and satiation of thirst and an interoceptordriven theory of origins of primary consciousness. Proceedings of the National Academy of Sciences, Washington, v. 96, n. 9, p. 5304-5309, 1999.

GAYTON, A.C.; HALL, J. E. Tratado de fisiologia médica. 10. ed. Rio de Janeiro: Guanabara Koogan, 2002.

GOIS, C. F. L.; AGUILLAR, M. O.; SANTOS, V.; RODRÍGUEZ, E. O. L. Fatores estressantes para o paciente submetido a cirurgia cardíaca. Investigación y Educación en Enfermería, Medellín, v. 30, n. 3, p.312-9, 2012. Disponível em: $<$ http://www.scielo.org.co/scielo.php?script=sci arttext\&pid $=$ S0120-53072012000300003\&lng $=\bar{p}$ t\&nrm=iso $>$. Acesso em: 5 abr. 2014.

HUMPHREYS, J.; DONESKY, D. A.; DRACUP, K.; LEE, K. A.; AOUIZEREAL, B.; MIASKOWSKI, C.; BAGGOTT, C.; BARGER, M.; FRANCK, L.; KENNEDY, C.; CARRIERIKOHLMAN, V.; PUNTILLO, K.; FAUCETT, J.; JANSON, S. Theory of symptom management. In: SMITH, M. J.; LIEHR, P. R. (Ed.). Middle range theory for nursing. 2nd New York: Springer Publishing Company, 2008. p. 145-58.

HUR, Y. S.; SHIN, K. A.; LEE, W. J.; LEE, J. O.; IM, H. J.; KIM, Y. M. The comparison of moisturizing effect of cold water gargling, wet gauze application and humidification in reducing thirst and mouth dryness after nasal surgery. Journal of Korean Clinical Nursing Research, v. 15, n. 1, p. 43-53, 2009.

LEIPER, J. Thirst. In: CABALLERO, B.; ALLEN, L.; PRENTICE, A. Encyclopedia of human nutrition. 2nd. Aberdeen, UK: Elsevier Academic Press, 2005. p. 278-286.
MILLARD-STAFFORD, M.; WENDLAND, D.; O'DEA, N. K.; NORMAN, T. L. Thirst and hydration status in everyday life. Nutrition Reviews, Washington, v. 70, n. 2, p. 147-51, 2012.

NASCIMENTO, L. A.; FONSECA, L. F. Sede do paciente cirúrgico: elaboração e validação de um protocolo de manejo seguro da sede. Revista de Enfermagem UFPE online, Recife, v. 7, p. 1055-8, mar. 2013.

PASQUALI, L. Testes referentes a construto: teoria e modelo de construção. In: PASQUALI, L. Instrumentos psicológicos: manual prático de elaboração. Brasília: LABPAM/IBAPP, 1999. p. 37-72.

ROLLS, B. J.; WOOD, R. J.; ROLLS, E. T.; LIND, H.; LIND, W.; LEDINGHAM, J. G. G. Thirst following water deprivation in humans. American Journal of Physiology, Washington, v. 239, n. 5, p. 476-482, 1980.

WELCH, J. L. Development of the thirst distress scale. Nephrology Nursing Journal, Pitman, v. 29, n. 4, p. 337-41, 2002.

WESTMORELAND, D.; WESORICK, B.; HANSON, D.; WYNGARDN, K. Consensual validation of clinical practice model practice guidelines. Journal of Nursing Care Quality, Hagerstown, v. 14, n. 4, p. 16-27, 2000.

YOON, S. Y.; MIN, H. S. The effects of cold water gargling on thirst, oral cavity condition, and sore throat in orthopedics surgery patients. The Korean Journal of Rehabilitation Nursing, Korean, v. 14, n. 2, p. 136-44, 2011.

Recebido em: 11 jun. 2014. Aceito em: $06 \mathrm{fev} .2015$. 